\title{
Image Processing Methods for Quantitative Analysis of Mitochondrial DNA Dynamics
}

Toshiki Matsuo, Kyohei Nakayama, Kazuki Nishimoto and Yasutomo Nomura*

Department of Systems Life Engineering, Graduate School of Engineering, Maebashi Institute of Technology, Japan

\begin{abstract}
Mitochondria and mitochondrial DNA are known to be highly motile. Molecular analysis revealed that several proteins are bound between mitochondrial DNA and mitochondrial inner membrane. However, nucleoid formed by these proteins is involved in the replication and duplication of mitochondrial DNA and it remains unclear whether mitochondrial DNA keeps binding the mitochondrial inner membrane or not. When nucleoid is necessary during cell cycle, it would be formed probably. When nucleoid is formed in the manner dependent on mitochondrial DNA sequence, the changes in the sequence would result in the equilibrium shifted to dissociation. This implies that the important information on changes in mtDNA accompanied with various symptoms is obtained by quantitative analysis of mtDNA dynamics. In this review, theoretical background of two typical methods, i.e., particle trajectory and image correlation spectroscopy, and several results of the pilot experiment are introduced.
\end{abstract}

Keywords: Mitochondrial DNA; Particle trajectory; Image correlation spectroscopy; Diffusion constant; Mean squared displacement

\section{Introduction}

Mitochondrion is one of major organelles within a cell. Mitochondria generate ATP when electron is transferred from respiratory substrates to oxygen by a series of redox reaction in which respiratory enzymes pump protons across the mitochondrial inner membrane from the matrix space [1]. In the isolated mitochondria as well as the intact cells, respiration frequently produces reactive oxygen species (ROS). Especially, ROS increases if respiration is perturbed, e.g., ischemia-reperfusion injury [2]. ROS can attack almost all biomolecules unspecifically. In case of DNA, ROS causes single and double-strand breaks, and base damage [3].

Mitochondrial DNA (mtDNA) that mitochondria contain independently of nucleus is particularly vulnerable because mtDNA repair system is weaker than that of nucleus [4]. As shown in Figure 1A, since mtDNA with $\sim 17 \mathrm{kbp}$ codes a part of respiratory enzymes [5], the damages would be harmful to the mitochondrial function. The damaged mtDNA has been reported to be involved in variety of human diseases, including diabetes, cancer, Parkinson's disease, and Alzheimer's disease $[4,6,7]$. Furthermore, mutation in mtDNA causes many diseases and mutations more than 50 have been identified [8,9]. Because the downstream consequences in humans cause the diverse and complex symptoms by impairing mitochondrial oxidative phosphorylation, the diagnosis is complicated. So far, the damaged mtDNA or mutation in mtDNA has been detected by using DNA purified from cells or tissues of the patients $[10,11]$. If the changes in mtDNA within living cells can be diagnosed noninvasively, the diagnosis process becomes simple and the effect of various pharmaceutical treatments on the cells would be estimated or further biochemical analysis can be possible.

Immunocytochemical studies suggested that mtDNA molecules were clustered within mitochondria as protein-DNA complexes called nucleoid and were bound to mitochondrial inner membrane [12]. Cell growth and proliferation require the continuous growth of cellular compartments, including the mitochondrial network. In contrast to nuclear DNA replication, mtDNA continues to be replicated and turned over in post-mitotic cells [13]. Furthermore, depending on the cell type, steady-state mtDNA copy number has been shown to vary, with highest levels being present in the most energy demanding tissues and in the ovum with an estimated copy number of up to 200,000 [14,15]. As shown in Figure 1B, each nucleoid contained several mtDNA copies. Among nucleoid proteins reported previously, main proteins were transcription factor A of mitochondria, TFAM [16], mitochondrial single-stranded DNA binding protein, mtSSB [17], mtDNA helicase, Twinkle [18], and mtDNA polymerase, POLG [12]. These proteins would participate in the maintenance of mtDNA. When a cell divides during cell cycle, daughter cells need to receive mtDNA. The transmission of the damaged mtDNA may differ from that of the intact mtDNA as discussed in heteroplasmy [19]. When nucleoid is formed in the manner dependent on mtDNA sequence, the changes in the sequence would result the equilibrium shifted to dissociation and would emphasize diffusion microscopically. Studies on living cells stained with bromdeoxyuridine (BrdU) have revealed an intramitochondrial distribution of mtDNA [20], as well as the capacity of mtDNA to diffuse into the mitochondria of rho (0) cells [21]. These imply that the important information on changes in mtDNA accompanied with various symptoms is probably obtained by quantitative analysis of mtDNA dynamics. In this review, theoretical background of the typical methods and several results of the pilot experiment are introduced.

\section{Image Aquisition}

When mtDNA dynamics has been analyzed, the standard

*Corresponding author: Dr. Yasutomo Nomura, Department of Systems Life Engineering, Graduate School of Engineering, Maebashi Institute of Technology, 460-1 Kamisadori, Maebashi, 371-0816, Japan, Tel: +81-27-265-7389; E-mail: ynomura@maebashi-it.ac.jp

Received January 05, 2012; Accepted February 24, 2012; Published February 26, 2012

Citation: Matsuo T, Nakayama K, Nishimoto K, Nomura Y (2012) Image Processing Methods for Quantitative Analysis of Mitochondrial DNA Dynamics. Biochem \& Anal Biochem S3-001. doi:10.4172/2161-1009.S3-001

Copyright: (C) 2012 Matsuo T, et al. This is an open-access article distributed under the terms of the Creative Commons Attribution License, which permits unrestricted use, distribution, and reproduction in any medium, provided the original author and source are credited. 
fluorescence imaging systems (e.g., laser scanning microscope, LSM) are adopted [22]. As shown in Figure 2A, the high quality image data were obtained with FluoView FV1000 Olympus and were used in this paper. HEK293 cells were incubated in 300x diluted PicoGreen reagent (Molecular Probes) under culture conditions for $1 \mathrm{hr}$. Since DNA intercalater PicoGreen was used, mtDNA in cytoplasm and nuclear DNA were observed [23]. Ashley et al. [23] pointed out that PicoGreen staining of mtDNA was dependent on a mitochondrial membrane potential. This contributes to the fluorescence of mtDNA stronger than that of nuclear DNA within a living cell in contrast to DNA solution. In order to analyze mtDNA dynamics, an area of cytoplasm was selected (Figure 2B) and the time image series was recorded. Each image series (256 x 256 pixels $\times 30$ frames in the case of particle trajectory, 100 frames in image correlation spectroscopy) with $10 \mathrm{~s}$ between sequential frames was collected from single cells.

\section{Diffusion Equation}

If mtDNA assumes to diffuse freely, the first step for the analysis is to derive diffusion equation. The equation is usually given as follows [24]:

$$
\frac{\partial c}{\partial t}=D \frac{\partial^{2} c}{\partial x^{2}}
$$

Where $c$ indicates the concentration at the point $x$ and time $t$. $D$ represents the diffusion constant. When the linear partial differential equation of second order is solved analytically by using Dirac delta function as initial conditions, the following equation is obtained:

$$
c(x, t)=\frac{1}{\sqrt{4 \pi D t}} e^{-\frac{x^{2}}{4 D t}} .
$$

Using Einstein-Stokes equation, $D$ is as follows:

$$
D=\frac{R T}{6 \pi \eta N_{A}} \text {. }
$$

A

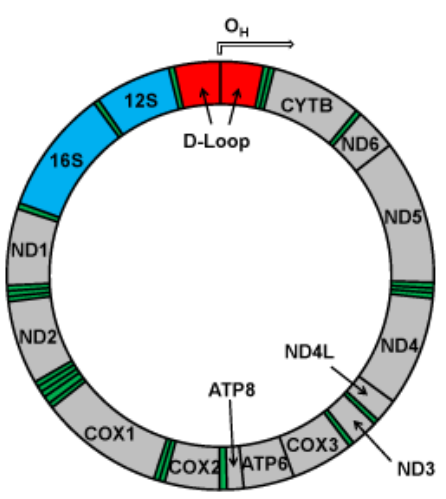

B

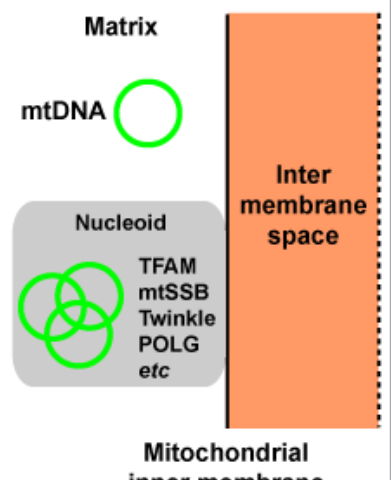

inner membrane
Figure 1: Structure of circular mitochondrial DNA (mtDNA). A: The human mitochondrial genome $(\sim 17 \mathrm{kbp})$ encodes 13 subunits of respiratory enzymes which are indicated by areas filled with grey: NADH dehydrogenase (ND 1-6), cytochrome b (CYTB), cytochrome oxidase (COX 1-3), FoF1 ATP synthase (ATP6 and 8). MtDNA also encodes $12 \mathrm{~S}$ and 16S rRNA genes which are indicated by areas filled with blue and 22 tRNA genes filled with green. Replication of the mtDNA occurs in the D-loop region which is indicated by areas filled with red. $\mathrm{O}$ is the replication origin of the heavy chain (NCBI Reference Sequence: NC_012920.1). B: Free mtDNA and nucleoid. Several mtDNA molecules within a nucleoid associate with mitochondrial inner membrane through the complex formed with nucleoid proteins such as TFAM, mtSSB, Twinkle, POLG and so on.
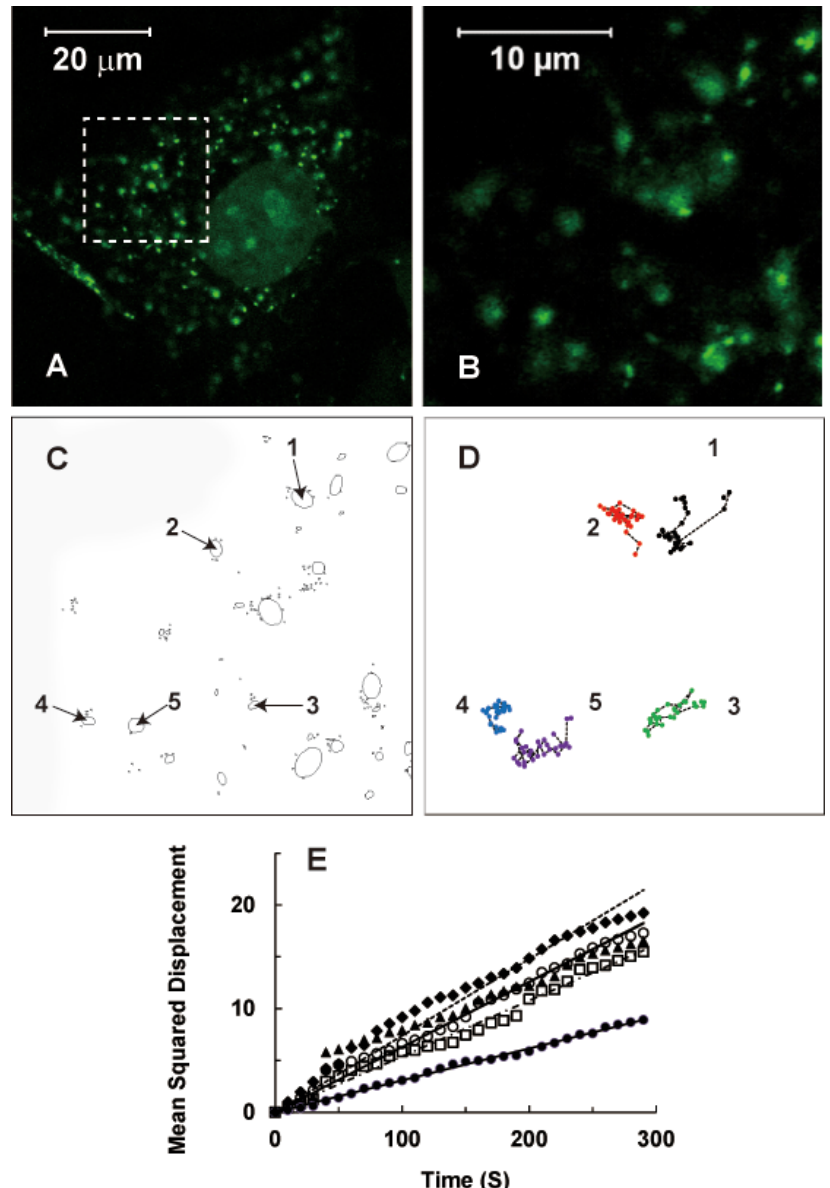

Figure 2: Particle trajectory method. A: Confocal image of single cell stained with DNA intercalater PicoGreen. A large nucleus in almost center area within a cell and bright points in the cytoplasm can be observed. B: The area marked by a dotted square in $A$ is enlarged for recording a time image series. C: Particles of mtDNA was extracted from each frame in the time image series through the setting proper threshold. Five particles used in this study are numbered from 1 to 5. D: Trajectories of center points of particles obtained by fitting ellipses. Analyzed particles are the same as C. E: Relationship between mean squared displacement and time. Particle \#1 in $C$ denotes closed triangle and the linear regression shows thin solid line, \#2: open circle and thick solid line, \#3: open square and chain line, \#4: closed circle and solid line, \#5: closed diamond and dotted line.

where $R, T, \eta$, and $N_{A}$ are the gas constant, the absolute temperature, viscosity and Avogadro number, respectively. Because $\eta$ is pointed out to be spatially heterogeneous within a cell, the value should be considered carefully $[25,26]$. In this paper, two methods to analyze dynamics of mtDNA which diffuse freely within mitochondria are outlined, $(i)$ trajectory method of fluorescent particles selected in temporally serial images [27-31] and (ii) image correlation spectroscopy using the entire images [32,33].

\section{Particle Trajectory Method}

In the particle trajectory method of fluorescent mtDNA, the image is quiet frequently processed by a public domain Java image processing program, e.g., ImageJ. In this paper, first of all, particles were extracted from background in the time image series by the means of setting threshold level (Figure 2C) and the center points of particles were calculated by best fitting to ellipses. The center points were tracked in the time image series (Figure 2D). 
Diffusion constant is calculated from tracking data of particles. If the particles move from position 0 at time 0 to new position $x$ after time $t$ due to the simple diffusion, mean squared displacement (MSD) is given by integral using probability distribution of Equation (2) [24]:

$$
\left\langle x^{2}\right\rangle=\int_{-\infty}^{\infty} x^{2} \cdot \frac{1}{\sqrt{4 \pi D t}} \exp \left(-\frac{x^{2}}{4 D t}\right) d x=2 D t .
$$

MSD is $4 D t$ in the case of two dimensions (2D) and $6 D t$ in three dimensions (3D). Equation (4) indicates that MSD increases with time linearly. Indeed, Figure 2E indicates linear relationship between time and MSD calculated from Figure 2D. Each slope of Figure 2D shows diffusion constant of five mtDNAs, and data of 4 cells are summarized in Table 1. Although diffusion constant of $20 \mathrm{mtDNA}$ particles from 4 cells was $9.8+5.5 \mu \mathrm{m}^{2} / \mathrm{s}$ (mean $+\mathrm{SD}$ ), careful interpretations for the data were required. When the actual 3D dynamics of mtDNA within a cell was observed by using LSM, 2D serial images with $x$ axis and $y$ axis were recorded with time but contained no information about $z$ axis. Therefore, since a true diffusion constant in 3D space was not calculated, the apparent value was comparable with two thirds of the diffusion constant. Although the calculation of temporal series of 3D reconstructions from $2 \mathrm{D}$ image stacks is not always impossible, the reconstruction is the computationally intense task and it would be not employed practically. Although diffusion coefficient of mtDNA in single living cell was two digits smaller than that of them from in vitro $[34,35]$ careful considerations about the spatially heterogeneous viscosity within a cell would be required.

\section{General Expression of Image Correlation Spectroscopy (ICS)}

In correlation analysis, the cross-correlation of a signal with itself is calculated and the similarity between signals observed as a function of time or space is obtained [32]. A generalized spatiotemporal correlation function is defined as following equation:

$$
r(\xi, \eta, \tau)=\frac{\langle\delta I(x, y, t) \cdot \delta I(x+\xi, y+\eta, t+\tau)\rangle}{\langle I(x, y, t)\rangle^{2}} .
$$

Where a fluctuation in fluorescence, $\delta I(x, y, t)$, is given as follows:

$$
\delta I(x, y, t)=I(x, y, t)-\langle I(x, y, t)\rangle .
$$

Where $I(x, y, t)$ is the intensity at pixel $(x, y)$ in the image recorded at time tin image series as shown in Figure 3A. $\langle I(x, y, t)\rangle$ is the average intensity of that image at time $t$. Every image obtained by LSM is a convolution of the microscope PSF (point spread function) with the point source emission from the fluorescent particle due to diffraction [36]. This convolution causes the signal from a point-emitter to be spread over a number of pixels. Dynamics of the fluorescent particle can be calculated by correlating fluorescence fluctuation signals arising from the particles entering and exiting observation area defined by the microscope PSF.

\section{Spatial ICS}

To obtain the microscope PSF, fluorescence fluctuations over plane of each image are correlated from Equation (5):

$$
r(\xi, \eta, 0)=\frac{\langle\delta I(x, y, t) \cdot \delta I(x+\xi, y+\eta, t)\rangle}{\langle I(x, y, t)\rangle^{2}} .
$$

The correlation function is then fitted to a 2D Gaussian using a nonlinear least squares algorithm:
A
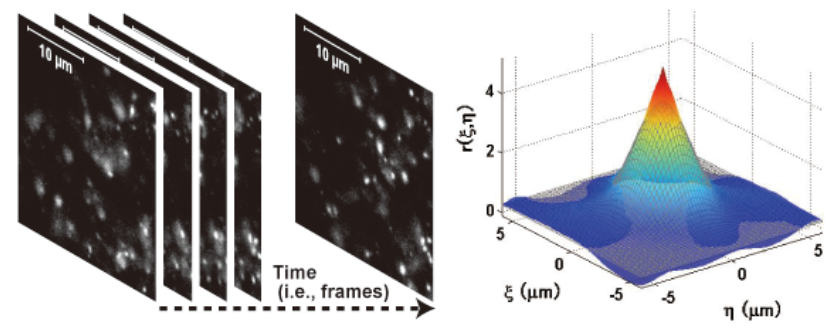

C
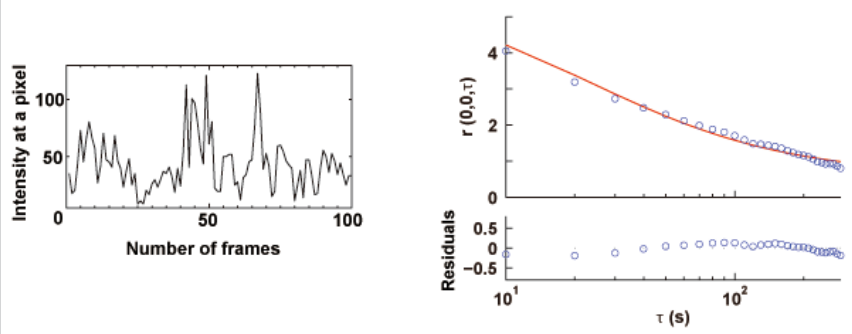

Figure 3: Image correlation spectroscopy. A: Several frames of time image series of Figure 2B. B: Spatial ICS to obtain the point spread function of LSM (see text). For example, the raw spatial autocorrelation function of the 1st frame of the image series is denoted by the colored surface, and the fitted $2 \mathrm{D}$ Gaussian function is denoted by the grey mesh. The point spread function, i.e., beam radius of LSM, is obtained by the fitting. C: Intensity at a pixel fluctuates with number of frames separated $10 \mathrm{~s}$ interval. D: Temporal ICS to obtain the diffusion time in which mtDNAs transverse the beam radius. A temporal image autocorrelation function (open circles) was derived from the time image series same as A. The temporal autocorrelation function was fitted to a simple one component $2 \mathrm{D}$ model (solid line) which can be derived from the diffusion equation. The residuals are denoted in the bottom. Finally, diffusion constant was calculated from the beam radius and diffusion time as shown in Table 1.

$$
r(\xi, \eta, 0)=g(0,0,0) \cdot \exp \left[-\frac{\xi^{2}+\eta^{2}}{\omega_{0}^{2}}\right]+g_{\infty} .
$$

Where $g(0,0,0)$ is the zero-lags amplitude and is inversely proportional to the number of fluorescent particles, and $g_{\infty}$ is the longspatial lag offset to account for an incomplete decay of the correlation function. In Equation (8), a Gaussian function is used because the laser beam has a Gaussian intensity profile. A typical result is shown in Figure $3 \mathrm{~B}$. The beam radius of the microscope PSF $\left(\omega_{0}\right)$ can be confirmed by using methods such as imaging of fluorescent microspheres with diameter less than diffraction limit.

\section{Temporal ICS}

Because the observation area is defined as $\omega_{0}$ in the preceding section, dynamics of fluorescent particles is calculated from temporal fluctuation of fluorescence intensity in the area (Figure 3C). As shown in Figure 3D, temporal autocorrelation function of an image series as a function of time lag $\tau$ is obtained from Equation (5) when $\xi$ and $\eta=0$ :

$$
r(0,0, \tau)=\frac{\langle\delta I(x, y, t) \cdot \delta I(x, y, t+\tau)\rangle}{\langle I(x, y, t)\rangle^{2}} .
$$

Where the angular brackets denote spatial and temporal averaging

Experimentally, $\tau$ values are determined by the time between subsequent images in the image series. Depending on the microscope system used, sampling time of image acquisition is usually between 


\begin{tabular}{|c|c|c|}
\hline & Trajectory & ICS \\
\hline Diffusion constant & $9.8+5.5$ & $9.4+10.2$ \\
\hline$\left(\times 10-3 \mu \mathrm{m}^{2} / \mathrm{s}\right)$ & $(\mathrm{n}=4$ cells $)$ & $(\mathrm{n}=25$ cells $)$ \\
\hline
\end{tabular}

Table 1: Diffusion constant of mtDNA evaluated by two analysis methods.

0.03 and $10 \mathrm{~s}$ and was 10 sin this paper. Here, the correlation function $r(0,0, \tau)$ is fitted to a simple one component $2 \mathrm{D}$ model which can be derived from Equation (2):

$$
r(0,0, \tau)=\frac{g(0,0,0)}{\left(1+\frac{\tau}{\tau_{d}}\right)}+g_{\infty} .
$$

Where $g(0,0,0)$ is the zero-lags amplitude dependent on number of fluorescent particles, and $g_{\infty}$ is the long-time offset. For confocal excitation, the characteristic diffusion time, $\tau_{d}$ is related to the diffusion coefficient, $D$ by:

$$
D=\frac{\left\langle\omega_{0}\right\rangle^{2}}{4 \tau_{d}} .
$$

Where $\omega_{0}$ is $e^{-2}$ radius of the focused beam of the microscope

As shown in Table 1, diffusion constant of mtDNA particles from 25 cells was $9.4+10.2 \mu \mathrm{m}^{2} / \mathrm{s}$ (mean $\left.+\mathrm{SD}\right)$. Although this average value was comparable with that of the particle trajectory method, SD was larger than that of trajectory method. This difference may be resulted from exclusion of extraordinary movement during particle selection. In Equation (8), a 2D simple diffusion model is adopted for comparison with results obtained by the particle trajectory method, but 3D simple diffusion model should be used in the next step.

\section{Concluding Remarks and Future Prospects}

In this paper, typical two methods to analyze mtDNA dynamics were explained concisely. At present, it is difficult to compare between the particle trajectory method and the image correlation spectroscopy quantitatively because of the different number of cells analyzed. However, we try to apply particle trajectory method to more cells. The reason of the standard deviation less than results of image correlation spectroscopy would attribute to the selection of the several particles which were observable easily. If we select all particles in the time image series in the case of particle trajectory method as same as image correlation spectroscopy, the standard deviation would be comparable to that of image correlation spectroscopy. The merit of image correlation spectroscopy is that one can analyze the average diffusion constant for all particles without the complicated procedures of the selection of particles. Thus, because the method would be effective in an automated diagnosis for mitochondrial diseases, this method is expected to be applied to various sample containing patients suspected of having these diseases. At present, we plan to determine the effect of mtDNA damage on the diffusion constant in detail. Furthermore, because mitochondria within a cell move through the interaction with cytoskeleton, the effects of the cytoskeleton inhibitors on mtDNA dynamics may be quite interesting.

\section{Acknowledgments}

This study was financially supported in part by Grant-in-Aid for Scientific Research (C) (23500523) and Knowledge Cluster Initiative (Hakodate Marine BioIndustrial Cluster) from the Ministry of Education, Science, Sports and Culture of Japan.

\section{References}

1. Santos JH, Hunakova L, Chen Y, Bortner C, Van Houten B (2003) Cell sorting experiments link persistent mitochondrial DNA damage with loss of mitochondrial membrane potential and apoptotic cell death. J Biol Chem 278: 1728-1734.

2. Das DK, Maulik N (2003) Preconditioning potentiates redox signaling and converts death signal into survival signal. Arch Biochem Biophys 420: 305-311.

3. Demple B, Harrison L (1994) Repair of oxidative damage to DNA: enzymology and biology. Annu Rev Biochem 63: 915-948.

4. Yakes FM, Van Houten B (1997) Mitochondrial DNA damage is more extensive and persists longer than nuclear DNA damage in human cells following oxidative stress. Proc Natl Acad Sci U S A 94: 514-519.

5. Wallace DC (1999) Mitochondrial diseases in man and mouse. Science 283: 1482-1488.

6. Chinnery PF, DiMauro S, Shanske S, Schon EA, Zeviani M, et al. (2004) Risk of developing a mitochondrial DNA deletion disorder. Lancet 364: 592-596.

7. Driggers WJ, LeDoux SP, Wilson GL (1993) Repair of oxidative damage within the mitochondrial DNA of RINr 38 cells. J Biol Chem 268: 22042-22045.

8. Chen TJ, Boles RG, Wong LJ (1999) Detection of mitochondrial DNA mutations by temporal temperature gradient gel electrophoresis. Clin Chem 45: 11621167.

9. Wong LJ (2004) Comprehensive molecular diagnosis of mitochondrial disorders: qualitative and quantitative approach. Ann N Y Acad Sci 1011: 246258.

10. Deschauer M, Krasnianski A, Zierz S, Taylor RW (2004) False-positive diagnosis of a single, large-scale mitochondrial DNA deletion by Southern blot analysis: the role of neutral polymorphisms. Genet Test 8: 395-399.

11. Richter C, Park JW, Ames BN (1988) Normal oxidative damage to mitochondrial and nuclear DNA is extensive. Proc Natl Acad Sci U S A 85: 6465-6467.

12. Bogenhagen DF, Rousseau D, Burke $S$ (2008) The layered structure of human mitochondrial DNA nucleoids. J Biol Chem 283: 3665-3675.

13. Magnusson J, Orth M, Lestienne P, Taanman JW (2003) Replication of mitochondrial DNA occurs throughout the mitochondria of cultured human cells. Exp Cell Res 289: 133-142.

14. Moraes CT (2001) What regulates mitochondrial DNA copy number in animal cells? Trends Genet 17: 199-205.

15. Shoubridge EA (2000) Mitochondrial DNA segregation in the developing embryo. Hum Reprod 15 Suppl 2: 229-234.

16. Alam TI, Kanki T, Muta T, Ukaji K, Abe Y, et al. (2003) Human mitochondrial DNA is packaged with TFAM. Nucleic Acids Res 31: 1640-1645.

17. Maier D, Farr CL, Poeck B, Alahari A, Vogel M, et al. (2001) Mitochondrial singlestranded DNA-binding protein is required for mitochondrial DNA replication and development in Drosophila melanogaster. Mol Biol Cell 12: 821-830.

18. Spelbrink JN, Li FY, Tiranti V, Nikali K, Yuan QP, et al. (2001) Human mitochondrial DNA deletions associated with mutations in the gene encoding Twinkle, a phage T7 gene 4-like protein localized in mitochondria. Nat Genet 28: 223-231.

19. Chinnery PF, Thorburn DR, Samuels DC, White SL, Dahl HM, et al. (2000) The inheritance of mitochondrial DNA heteroplasmy: random drift, selection or both? Trends Genet 16: 500-505.

20. Legros F, Malka F, Frachon P, Lombès A, Rojo M (2004) Organization and dynamics of human mitochondrial DNA. J Cell Sci 117: 2653-2662.

21. Hayashi J, Takemitsu M, Goto Y, Nonaka I (1994) Human mitochondria and mitochondrial genome function as a single dynamic cellular unit. J Cell Biol 125: 43-50.

22. Nomura Y, Takayama T, Sato M, Takahashi E, Feng Z, et al.(2009) Mitochondria-targeted green fluorescent protein for quantitative monitoring of mitochondrial morphology in living cells. Lett Drug Design Discov 6: 319-322.

23. Ashley N, Harris D, Poulton J (2005) Detection of mitochondrial DNA depletion in living human cells using Pico Green staining. Exp Cell Res 303: 432-446. 
Citation: Matsuo T, Nakayama K, Nishimoto K, Nomura Y (2012) Image Processing Methods for Quantitative Analysis of Mitochondrial DNA Dynamics. Biochem \& Anal Biochem S3-001. doi:10.4172/2161-1009.S3-001

Page 5 of 5

24. Einstein A (1956) Investigations on the theory of the Brownian movement. Dover publications, Mineola, New York.

25. Baumann G, Place RF, Földes-Papp Z (2010) Meaningful interpretation of subdiffusive measurements in living cells (crowded environment) by fluorescence fluctuation microscopy. Curr Pharm Biotechnol 11: 527-543.

26. Weiss $M$ (2008) Probing the interior of living cells with fluorescence correlation spectroscopy. Ann N Y Acad Sci 1130: 21-27.

27. Caspi A, Granek R, Elbaum M (2002) Diffusion and directed motion in cellular transport. Phys Rev E Stat Nonlin Soft Matter Phys 66: 011916.

28. Golding I, Cox EC (2006) Physical nature of bacterial cytoplasm. Phys Rev Lett 96: 098102.

29. Platani M, Goldberg I, Lamond Al, Swedlow JR (2002) Cajal body dynamics and association with chromatin are ATP-dependent. Nat Cell Biol 4: 502-508.

30. Seisenberger G, Ried MU, Endress T, Büning H, Hallek M, et al. (2001) Realtime single-molecule imaging of the infection pathway of an adeno-associated virus. Science 294: 1929-1932.
31. Selhuber-Unkel C, Yde P, Berg-Sørensen K, Oddershede LB (2009) Variety in intracellular diffusion during the cell cycle. Phys Biol 6: 025015.

32. Kolin DL, Wiseman PW (2007) Advances in image correlation spectroscopy: measuring number densities, aggregation states, and dynamics of fluorescently labeled macromolecules in cells. Cell Biochem Biophys 49: 141-164.

33. Nomura Y (2011) Direct Quantification of Mitochondria and Mitochondrial DNA Dynamics. Curr Pharm Biotechnol .

34. Nomura Y, Fuchigami H, Kii H, Feng Z, Nakamura T, et al. (2006) Detection of oxidative stress-induced mitochondrial DNA damage using fluorescence correlation spectroscopy. Anal Biochem 350: 196-201.

35. Nomura Y, Fuchigami H, Kii H, Feng Z, Nakamura T, et al. (2006) Quantification of size distribution of restriction fragments in mitochondrial genome using fluorescence correlation spectroscopy. Exp Mol Pathol 80: 275-278.

36. Yoo H, Song I, Gweon DG (2006) Measurement and restoration of the poin spread function of fluorescence confocal microscopy. J Microsc 221: 172-176 